\title{
Partial substitution of CdS buffer with interplay of fullerenes in kesterite solar cells.
}

Received 00th January 20xx, Accepted 00th January 20xx DOI: $10.1039 / x 0 \times x 00000 x$

\author{
David Payno, ${ }^{a}$ Yudania Sánchez $^{\mathrm{b}}$, Oriol Blázquez ${ }^{\mathrm{b}}$, Sergio Giraldo ${ }^{\mathrm{b}}$, Edgardo Saucedo ${ }^{\mathrm{b}, \mathrm{c}}$ and \\ Shahazada Ahmad ${ }^{a}$
}

Over the last decades, significant progress has been made in inorganic materials to enable them as next generation photovoltaic materials that can fulfil the green energy requirements. Cu2ZnSn(S,Se)4 stands out as a p-type absorber material due to exemption from scarce and strategic elements and its similarities with Cu2 InGa(S,Se)4. Organic materials based on fullerenes based derivates are effective $n$-type photovoltaic materials. We report the usage of $n$-type fullerenes materials with kesterite-based absorbers in a thin film, polycrystalline solar cell by a partial substitution of the CdS buffer layer with C60 and C70 fullerenes. Impedance measurements reveals that using C60 as an interlayer improve built-in potential, reduces the interface recombination and promote the charge conduction, resulting in an increase in the open circuit voltage and thus device performance.

\section{Introduction}

$\mathrm{Cu}_{2} \mathrm{ZnSn}(\mathrm{S}, \mathrm{Se})_{4}$ (CZTSSe) having a kesterite structure is a promising absorber material for large-scale solar integration, due to its unique composition constituted of abundant and nontoxic elements. It has a very high absorption coefficient, allowing it use on thin films and flexible devices, and a tuneable band-gap from $1-1.5 \mathrm{eV}$, a suitable range for a single junction, along with robust stability in external conditions. ${ }^{1}$ An added value of being solution processable makes it attractive even for small scale manufacturing. ${ }^{2}$ However, the progress in highly efficient devices based on Kesterite has been stuck and less explored during last years owing to its complexity and low power conversion efficiency as compared to the other thin film emerging photovoltaic technologies. ${ }^{3}$ One of the reasons for the lower performance is in the formation of the junction with the buffer layer. ${ }^{4}$ Unfavourable band alignment, dipole formation and lattice mismatch creates recombination paths that lower open-circuit voltage, while buffer layers, by absorbing part of the spectra, reduces the short-circuit current. CdS is the most studied n-type buffer layer in thin film solar cells, inherited from the established technologies of $\mathrm{CdTe}$ and $\mathrm{Cu}_{2} \operatorname{lnGa}(\mathrm{S}, \mathrm{Se})_{4}$, where it has shown competitive efficiencies, owing to its high transparency, electrical properties and ease of processing through chemical bath deposition (CBD). ${ }^{4-6}$ Attempts to modify or substitute this buffer layer was limited to $\mathrm{Zn}(\mathrm{S}, \mathrm{O})^{7-9}, \ln _{2} \mathrm{~S}_{3} 10$,

a. BCMaterials, Basque Center for Materials, Applications andNanostructures, BId. Martina Casiano, UPV/EHU Science Park, Barrio Sarriena s/n, 48940 Leioa, Spain

b. Catalonia Institute for Energy Research (IREC), Jardin de les Dones de Negre 1, 08930 Sant Adrià de Besòs, Barcelona, Spain.

c. Department of Electronic Engineering, Polytechnic University of Catalonia (UPC) Sor Eulàlia d'Anzizu 6, 08034 Barcelona, Spain.

Electronic Supplementary Information (ESI) available: [details of any supplementary information available should be included here]. See DOI: 10.1039/x0xx00000x
$(\mathrm{Zn}, \mathrm{Sn}) \mathrm{O}{ }^{11},(\mathrm{Cd}, \mathrm{Zn}) \mathrm{S}{ }^{12}$ or alloys with CdS 12,13 , but with low improvements in terms of reproducibility were obtained. In most of the cases, a second n-type material is required to improve the charge conduction and minimize short circuits, typically $\mathrm{ZnO}$, as window layer.

Organic semiconductors have delivered significant results, due to the possibility of tuneable electro-optical properties by engineering the molecules at synthesis steps. Fullerenes were being employed since $1985^{14}$, and their photovoltaic properties were first studied. ${ }^{15,16}$ Currently, fullerenes and its derivates are widely used in organic and perovskite based solar cells, as an efficient electron transport materials to achieve high performance. 17-19 Having advantages such as being environmentally friendly and stable, as they are composed of carbon with covalent bonds. They also can be functionalized to increase it solubility, like the more popular variant $[6,6]$-phenylC61-butyric acid methyl ester (PCBM).

However, reports dealing with fullerenes in kesterite solar cells are in scarce and limited efforts were made of combining CZTSSe with organic materials. CZTSSe nanocrystals have been used for photodetectors or improvements in organic solar cells ${ }^{20-22}$. Among the reports, Saha et al tried to create a planar superstrate device with CZTS nanoparticles and PCBM, with the limitation of having a non-ohmic contact between ITO and $\mathrm{CZTS}^{22}$, obtaining $0.9 \%$ power conversion efficiency (PCE), while Jang et al introduce CZTS nanoparticles into a bulk heterojunction (P3HT:PCBM) to improve the absorption of the organic solar cell, increasing the generated photocurrent, reaching a 3.32\% $\mathrm{PCE}^{21}$. Nevertheless, all these attempts lead to low performance devices.

In this work, we designed configurations for both CZTS and CZTSe kesterite based absorbers, by the introduction of n-type organic materials based on fullerenes $C_{60}$ and $C_{70}$ as interlayers and the reduction of $\mathrm{CdS}$ thickness. We settled for the best 
possible configuration of a double thin film buffer layer by changing the deposition order, and obtained promising results for both absorbers with $\mathrm{C}_{60}$. The open circuit voltage obtained are notably high with both absorbers, and gave a PCE of $8.37 \%$ and $4.58 \%$ with CZTSe and CZTS respectively. Additionally, an array of electrical measurements (impedance and C-V) were made for the CZTS samples to observe underlying phenomena, an increase in built in potential $\left(V_{b i}\right)$ and a reduction in the interface recombination was noted, allowing a significant improvement in the charge transport.

\section{Experimental method}

\section{Device}

For the fabrication of CZTSe and CZTS solar cells, a cleaned sodalime glass (SLG) where used as substrate and Na source. $800 \mathrm{~nm}$ thick film of Molybdenum (Mo) was sputtered on the SLG as back contact, followed by the deposition of metallic precursors $\mathrm{Sn}, \mathrm{Cu}$ and $\mathrm{Zn}$. For the selenium containing absorbers, also a thin film of $20 \mathrm{~nm}$ of Ge was deposit by thermal evaporation, as a doping agent to improve bulk quality ${ }^{23,24}$. This was then placed into a closed graphite box, and then introduced inside a quartz tube furnace to perform the sulfurization or selenization. The annealing treatment was performed in two steps, in a first step, low temperature was used to create the sulphides or selenides binary compounds, and in a second step high temperature to form the quaternary compound and to promote the grain growth.

An etching treatment was performed by introducing the samples on a $22 \%$ diluted $\left(\mathrm{NH}_{4}\right)_{2} \mathrm{~S}$ in $\mathrm{H}_{2} \mathrm{O}$, in order to remove the binary $\mathrm{Sn}(\mathrm{S}, \mathrm{Se})_{x}$ compounds formed at the interface. An extra air annealing treatment of $300^{\circ} \mathrm{C}$ was applied to the CZTS absorber to oxidize some of the remaining binary compounds.

The following buffer layers were deposited on top of the absorber. CdS was grown by chemical bath deposition (CBD). $\mathrm{Cd}\left(\mathrm{NO}_{3}\right)_{2}$ was used as $\mathrm{Cd}$ precursor of $\mathrm{CdS}$ in the $\mathrm{CBD}$, which allow for a better grow control of the thickness as the formation is slower ${ }^{25}$. A standard deposition of a $60 \mathrm{~nm}$ film in 40 minutes was required to achieve the best performance ${ }^{26,27}$, however, notably in this work, the process was reduced to 20 minutes in order to minimize the thickness, producing a thinner film of $\sim 30 \mathrm{~nm}$ of $\mathrm{CdS}$. The fullerenes $C_{60}$ and $C_{70}$ thin films were prepared following the procedure reported elsewhere ${ }^{18}$. The fullerenes were dissolved with a concentration of $10 \mathrm{mg} / \mathrm{mL}$ on 1,2-dichlorobenzene and keeping it stirring overnight. The solution was filtered with a PTFE filter of $0.42 \mu \mathrm{m}$ pore size, and deposited by spin coating $50 \mu \mathrm{L}$ at $1500 \mathrm{rpm}$ for 60 seconds. After spin coating, it was transferred on a hotplate to dry at $80^{\circ} \mathrm{C}$ for 2 minutes. The devices were finished by sputtering $50 \mathrm{~nm}$ of $\mathrm{ZnO}$ as window layer and $300 \mathrm{~nm}$ of ITO as transparent conductive film at $200^{\circ} \mathrm{C}$. No further, top metallic contact neither anti-reflection coating was used in this work. The cells were then mechanically scribed in squares of $3 \times 3 \mathrm{~mm}^{2}$.

\section{Characterization}

Absorption and transmittance spectre were recorded with the help of Agilent Cary 60 UV-Vis spectrometer. For the device characterization, the current-voltage curves the cells where measured on a class AAA Oriel solar simulator from Newport and a Keithley multi-meter, by connecting the positive contact to the exposed molybdenum and the negative directly to the ITO. The external quantum efficiency was measured on a PVE300 Photovoltaic EQE (IPCE) system, consisting on a $150 \mathrm{~W}$ Xenon lamp and a $1 / 4 \mathrm{~m}$ monochromator, in a range from 300 to $1100 \mathrm{~nm}$. For the impedance measurements, a constant bias signal with $20 \mathrm{mV}$ of perturbation was applied, in a frequency range from $5 \mathrm{GHz}-300 \mathrm{~Hz}$. They were taken inside a metallic box, to avoid interferences, with a Biologic impedance analyser, and EC-LAB software was used to make the fitting of the data to extract the parameters of the equivalent circuit.

\section{Results and discussion}

\section{Optical characterization}

A prerequisite of a buffer layer is high transmittance to a wide range of wavelengths, in order to allow the active material maximum light absorption. The optical properties were acquired on the films deposited on glass (Fig.1a).
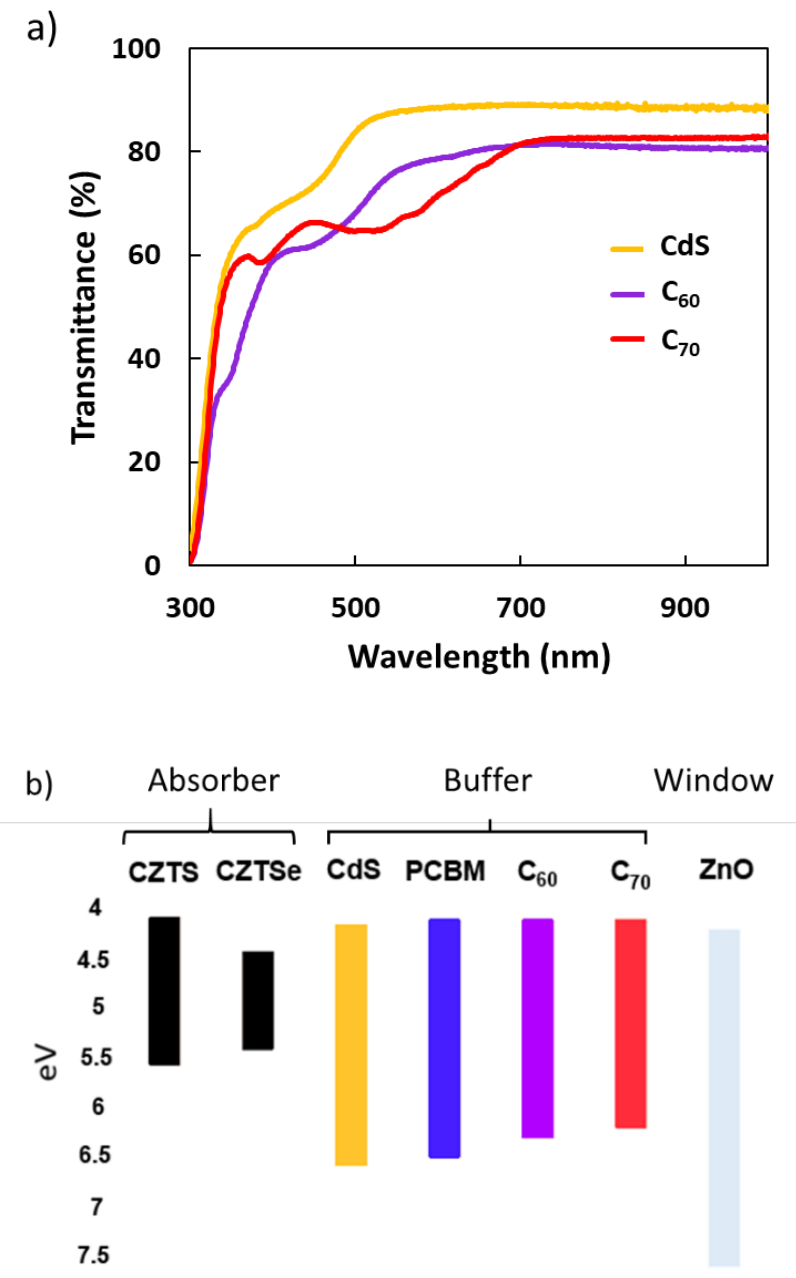

Figure 1. a) Transmittance spectra of thin films deposited on glass of $C_{60}$ and $C_{70}$ (spin coating) and CdS (CBD), and b) energy level diagram of the architect used (PCBM can be found on supporting information).

Transmittance spectra of the films represents a high transparency until 450nm wavelength for CdS and 500 nm for 
$\mathrm{C}_{60}$, while in the case of $\mathrm{C}_{70}$ it starts to show loss in transparency at $700 \mathrm{~nm}$. These transparency parameters suggest its usage as buffer layer with a low band-gap active material as kesterite. However, parasitic absorption can slightly reduce the photocurrent, effect that will be higher in the case of $C_{70}$. The optical band-gaps are calculated through a linear fit of the tauc plot (Fig.S1). The calculated values are in agreement with the typical values reported in the literature. Fig.1b illustrates the energy levels diagram of the device with the employed materials. In spite of different band-gaps of the materials, the energy level of the LUMO is close to the conduction band of the CZTS (e) in all cases, which facilitates the flow of electrons while the low energy level of the HOMO can block the holes effectively, making it ideal candidates as buffer layer.

\section{Device characterization}

Firstly, we have employed CZTSe as an absorber layer. Three different configurations were prepared (Fig.2) where CdS is reduced and partially substituted by a fullerene in a double buffer layer. The first one is denoted as $\mathrm{CdS} / \mathrm{C}_{60}$. In a second one $\mathrm{CdS} / \mathrm{C}_{70}$ was also probed as buffer film. In a third configuration, $\mathrm{C}_{60} / \mathrm{CdS}$ was used as interfacial agent, to test the possibility of reducing the interface recombination being deposited directly over the CZTSe absorber.

When pure selenide based kesterite was used, generally a post heat treatment (PHT) of the final device is performed to increase the efficiency. This treatment was found to improve the $p-n$ junction by allowing interdiffusion of elements such as $\mathrm{Cd}$ and Se for temperatures above $200 \circ \mathrm{C}$, reducing the interface recombination ${ }^{28}$. However, organic materials are susceptible to thermal treatments and can decomposed or oxidized at higher temperature. In our case, decomposition or melting was not an issue for fullerenes, as the covalent bonds of the carbon are extremely stable. ${ }^{29}$ However, it is vital to evaluate the role of organic layers under the required treatments and the possible effects this may impose in the fabricated solar cell, as changes in the crystal structure or oxidation may occur.

To evaluate this, the final devices were characterized before and after heat treatment of $230{ }^{\circ} \mathrm{C}$ for 20 minutes in air on a hotplate. The average parameters extracted from the $\mathrm{J}-\mathrm{V}$ characterization of eight different cells are showed in the Table 1. Similar trend was noted for all the devices with $C_{60}$. The $V_{o c}$ and the FF showed an increase after the temperature treatment, and gave an average PCE $>7.5 \%$. In the case of $C_{70}$, an improvement of the $V_{o c}$ and $J_{s c}$ can be noticed after the heat treatment, but the reduction in FF resulted in a drop in the final PCE. Table S1 illustrates a detailed study of post heat treatment and suggests the optimized results, with a treatment at $225 \circ \mathrm{C}$. The voltage produced can be further increased at higher temperatures, but a reduction of the current and $\mathrm{FF}$ will reduce the PCE. We can speculate that the use of organic layer is compatible with the required post heating treatments.

The devices using $\mathrm{C}_{60}$ between CZTSe and CdS showed a $V_{o c}$ of $20 \mathrm{mV}$ higher and competitive $\mathrm{FF}$ of $64 \%$ in average, that can indicate a surface passivation effect, but with a minute loss in photocurrent. The J-V curves (Fig.3a) of the best devices with post heat treatment yielded a value of $446 \mathrm{mV}$ of $V_{o c}$, without compromising other parameters, and a PCE of $8.37 \%$ was noted for the champion device. The $\mathrm{CdS} / \mathrm{C}_{60}$ also showed a similar value an PCE of $8.35 \%$ was obtained, due to a slightly higher photocurrent, but a reduced voltage. The external quantum efficiency (EQE) spectra of the devices (Fig. 3b), reveals that the loss of current is higher in the infrared region, which we assigned to the absorber layer itself rather than the buffer or the interfacial layers. Complementary to this, a higher response in the violet and ultraviolet region can be noticed (Fig. S3b), which can be attributed to a lower parasitic absorption of the double buffer layer in this region in comparison with a pure $\mathrm{CdS}$ buffer layer. On the other hand, a loss of response can be noticed in the infrared region generally, which is also responsible for the current lost from the ideal value. This drop in the infrared region is characteristic for CZTSe absorbers, and can be attributed to bulk defects that cause fast recombination of electron-hole pairs produced far from the $p-n$ junction.

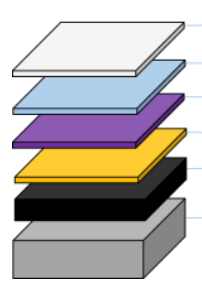

- Front contact ITO (Sputtering)

$\rightarrow$ Window ZnO (Sputtering)

$\rightarrow$ Fullerenes $\mathrm{C}_{60}$ or $\mathrm{C}_{70}$ (Spin coating)

Buffer CdS (CBD)

$\rightarrow$ Absorber CZTSe (Sputering)

$\rightarrow$ Molybdenum (Sputtering)

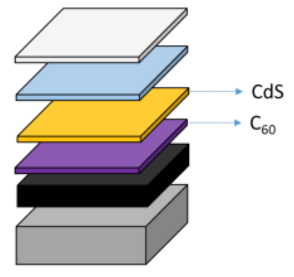

Figure 2. Architect of the different configurations of kesterites used for solar cells fabrication.

Table 1. Standard deviation of eight cells before and after the PHT.

\begin{tabular}{|c|c|c|c|c|c|}
\hline Type & $\begin{array}{l}\text { Post Heat } \\
\text { Treatment }\end{array}$ & $V_{\text {oc }}(\mathrm{mV})$ & $J_{\mathrm{sc}}\left(\mathrm{mA} / \mathrm{cm}^{2}\right)$ & $\mathrm{FF}(\%)$ & PCE (\%) \\
\hline \multirow{2}{*}{$\mathrm{CdS} / \mathrm{C}_{60}$} & As prepared & $404 \pm 5$ & $26.00 \pm 0.80$ & $\begin{array}{c}49.2 \pm \\
2.1\end{array}$ & $\begin{array}{c}5.16 \pm \\
0.30\end{array}$ \\
\hline & After PHT & $423 \pm 8$ & $27.91 \pm 0.73$ & $\begin{array}{c}63.7 \pm \\
3.5\end{array}$ & $\begin{array}{c}7.54 \pm \\
0.69\end{array}$ \\
\hline \multirow{2}{*}{$\mathrm{C}_{60} / \mathrm{CdS}$} & As prepared & $338 \pm 34$ & $24.24 \pm 2.2$ & $\begin{array}{c}41.0 \pm \\
5.6\end{array}$ & $3.45 \pm 1.1$ \\
\hline & After PHT & $443 \pm 6$ & $27.21 \pm 0.57$ & $\begin{array}{c}64.0 \pm \\
2.9\end{array}$ & $7.72 \pm 0.4$ \\
\hline \multirow[t]{2}{*}{$\mathrm{CdS} / \mathrm{C}_{70}$} & As prepared & $404 \pm 6$ & $19.62 \pm 0.80$ & $\begin{array}{c}31.7 \pm \\
1.1\end{array}$ & $\begin{array}{c}2.52 \pm \\
0.15\end{array}$ \\
\hline & After PHT & $416 \pm 2$ & $22.85 \pm 2.4$ & $\begin{array}{c}21.8 \pm \\
3.1\end{array}$ & $\begin{array}{c}2.09 \pm \\
0.48\end{array}$ \\
\hline
\end{tabular}

For the sample using $\mathrm{C}_{70}$, a drop in the FF led to a lower PCE than of $C_{60}$. The $V_{o c}$ remains similar, but there is a drop in the current value, as it was expected by transmittance data, and confirmed by the EQE measurement, where the signal is reduced for wavelengths shorter than the calculated bandgap (1.79 eV or $700 \mathrm{~nm})$. This behaviour is similar to the results of using $\mathrm{C}_{70}$ as buffer in other solar cell technologies ${ }^{30}$, but the plausible reasons of the low FF remains unclear, and we ascribed to a higher resistance of the $\mathrm{C}_{70}$ thin film. Fullerenes were also 
tested with CZTS absorber, using the similar three configurations. In this case, the surface passivation of the absorber is of significant interest, as the interface between CZTS and CdS presents several issues due to the valence band offset and secondary phases which induces interface recombination. Usually, an etching treatment ${ }^{31,32}$ or an oxide interlayer ${ }^{33,34}$ is required for a passivation of interface defects.
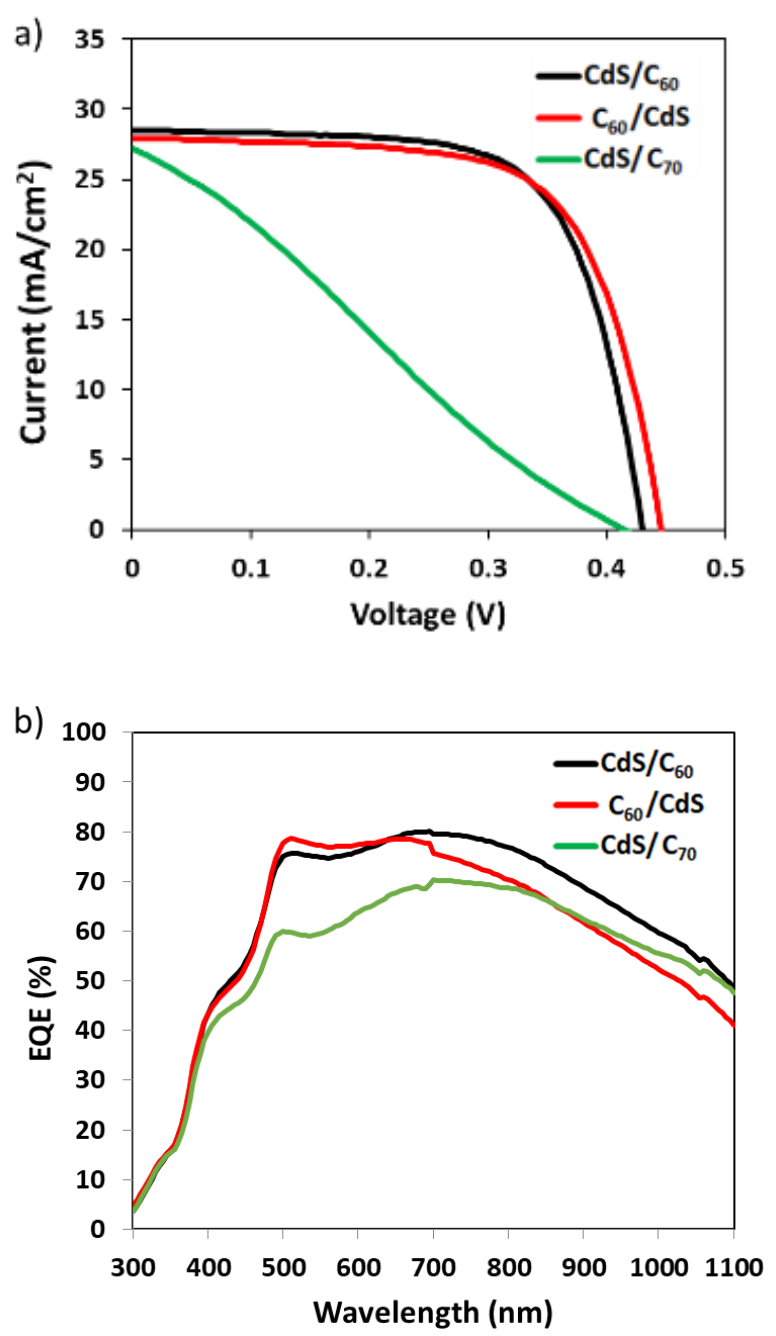

Figure 3. a) J-V curves under 1 sun illumination and b) EQE spectra of the best devices after the post heat treatment.

Figure 4 shows the $J-V$ curves of the best device for each condition under AM1.5 illumination condition, and the average values are showed in the Table 2 . In the case where $C_{60}$ was used as interlayer between the absorber and CdS, the devices experiment a significant increase in efficiency from $1 \%$ to $4.5 \%$, with a remarkable $V_{o c}$ of $718 \mathrm{mV}$ in the best cell, considering that the record value is $730 \mathrm{mV}$ for a pure CZTS absorber was achieved by a high temperature thermal annealing and a protective $\mathrm{Al}_{2} \mathrm{O}_{3}$ film in the back contact ${ }^{35}$. In this case, no special treatment was applied to the Molybdenum, so an excess of $\mathrm{MoS}_{2}$ forming during the sulfurization could increase the resistance and is responsible for the low FF in these devices.
The EQE spectra (Fig. 5a) was measured to investigate the deficiency of the current. No relevant changes in the band-gap of the absorber was noticed, having a value of $1.47 \mathrm{eV}$ for all cases. The spectra of the $\mathrm{CdS} / \mathrm{C}_{70}$ sample is losing part of the signal in the violet region, which can be associated again to the absorption of $\mathrm{C}_{70}$ on this region. However, the differences between the samples are smaller than expected from the $\mathrm{J}-\mathrm{V}$ and integrated photocurrent from the EQE is calculated (Fig.5b). The accumulated value should match with the $J_{s c}$ value b) measured on J-V curve but a difference from 6.24 and 7.27 $\mathrm{mA} / \mathrm{cm}^{2}$ was noted for the samples $\mathrm{CdS} / \mathrm{C}_{60}$ and $\mathrm{CdS} / \mathrm{C}_{70}$ respectively. This result reveals that there is a loss of photocurrent when they are illuminated under AM1.5 conditions, effect that is less noticed in the $\mathrm{C}_{60} / \mathrm{CdS}$ sample. This suggests change in the carrier concentration under light illumination. Light soaking is a common practice when CdS is used as buffer layer, which produces an increase of the electron density, increasing the conductivity and the width of the space charge region. In this case, this effect has a positive effect when the $\mathrm{C}_{60}$ is placed between CZTS and CdS, indicating that the electrons are transported efficiently through the buffer layer, but a barrier is created for the $\mathrm{CdS} / \mathrm{C}_{60}$ configuration, aggravating the accumulation and recombination of the charge in the interface, and explaining the discrepancy between the measured $J_{s c}$ and the integrated photocurrent in these cells. Table 2. Averages of the extracted parameters for each configuration with standard deviation

\begin{tabular}{ccccc}
\hline Condition & $V_{\text {oc }}(\mathrm{mV})$ & $J_{\mathrm{sc}}\left(\mathrm{mA} / \mathrm{cm}^{2}\right)$ & $\mathrm{FF}(\%)$ & PCE (\%) \\
$\mathrm{CdS} / \mathrm{C}_{60}$ & $520 \pm 8$ & $7.5 \pm 0.17$ & $27.8 \pm 0.6$ & $1.09 \pm 0.05$ \\
& & & & \\
$\mathrm{C}_{60} / \mathrm{CdS}$ & $700 \pm 32$ & $12.6 \pm 0.51$ & $46.7 \pm 2.9$ & $4.16 \pm 0.54$ \\
$\mathrm{CdS} / \mathrm{C}_{70}$ & $460 \pm 47$ & $5.3 \pm 0.33$ & $27.0 \pm 1.0$ & $0.65 \pm 0.07$ \\
\hline
\end{tabular}

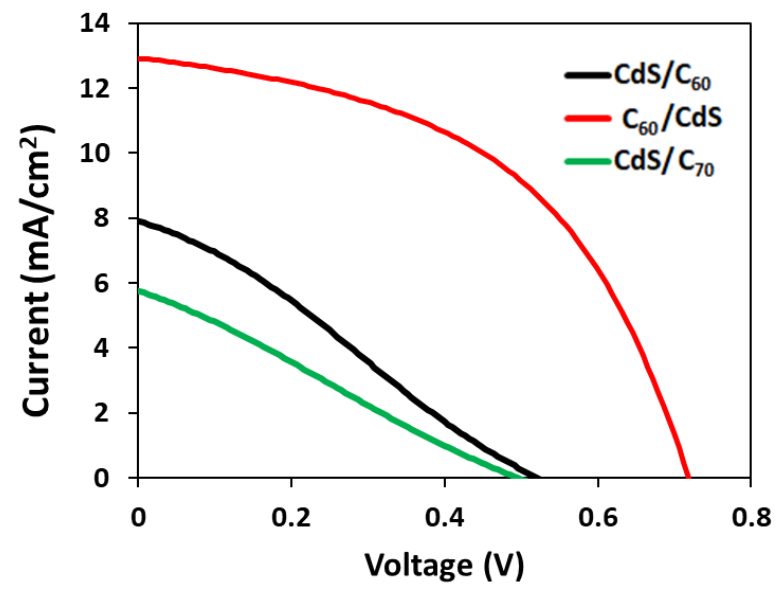

Figure 4. $J-V$ curves of the best cells using CZTS as absorber. 
a)

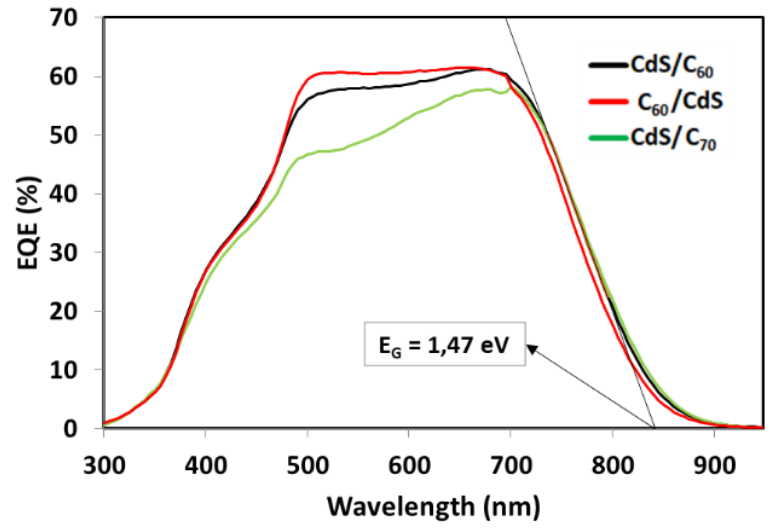

b)

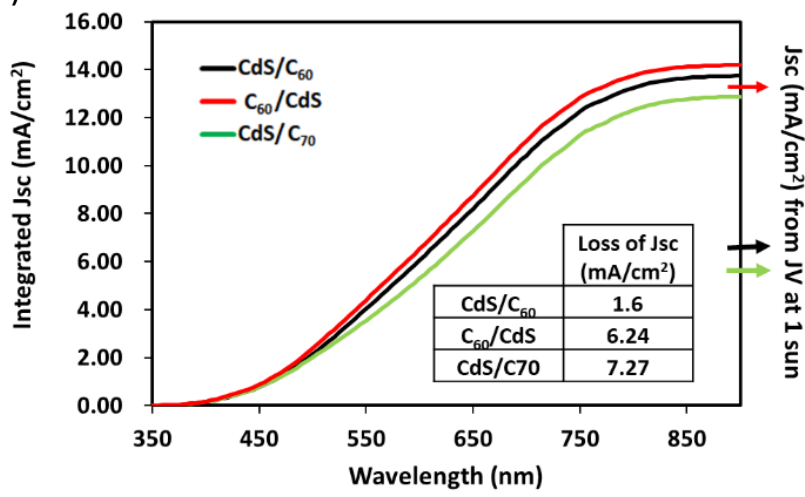

Figure 5. a) EQE spectra of the CZTS samples. The band-gap is calculated in the graph by a linear fitting of the last part of the graph and b) Corresponding integrated photocurrent density extracted from the EQE measurements. The inset table shows the difference between the integrated photocurrent and the $\mathrm{J}_{\mathrm{sc}}$ measured at 1 sun (marked with an arrow on the right).

\section{Impedance spectroscopy}

Impedance measurements were performed on the devices having a configuration $\mathrm{CZTS} / \mathrm{CdS} / \mathrm{C}_{60}$ and $\mathrm{CZTS} / \mathrm{C}_{60} / \mathrm{CdS}$ to elucidate the role of junction formed. The measure of the capacitance at different frequencies can display the behaviour of physical mechanisms occurring at different times scales. While the change of the applied voltage will modify carrier density and the space charge region. 3D mapping of the capacitance from a simple RC circuit is represented in the Fig.6a. The capacitance contribution mainly originates from the pseudo-capacitor formed by the $p-n$ junction between the absorber and the buffer layer, represented in the scheme (Fig.6b). Three main features can be distinguished:

i) At lower frequencies of $10^{2}-10^{4} \mathrm{~Hz}$, the capacitance rapidly increases with the increment of the direct voltage. While the capacitance is inversely proportional to the width of the depletion region according to the (Eq.1) of a capacitor, the width of the depletion region at this point is minimum. For an applied voltage higher than built-in potential, the $p-n$ junction allows pass of the current and the capacitance is dominated by the carrier diffusion, which is proportional to the current, as show in the (Eq.2).

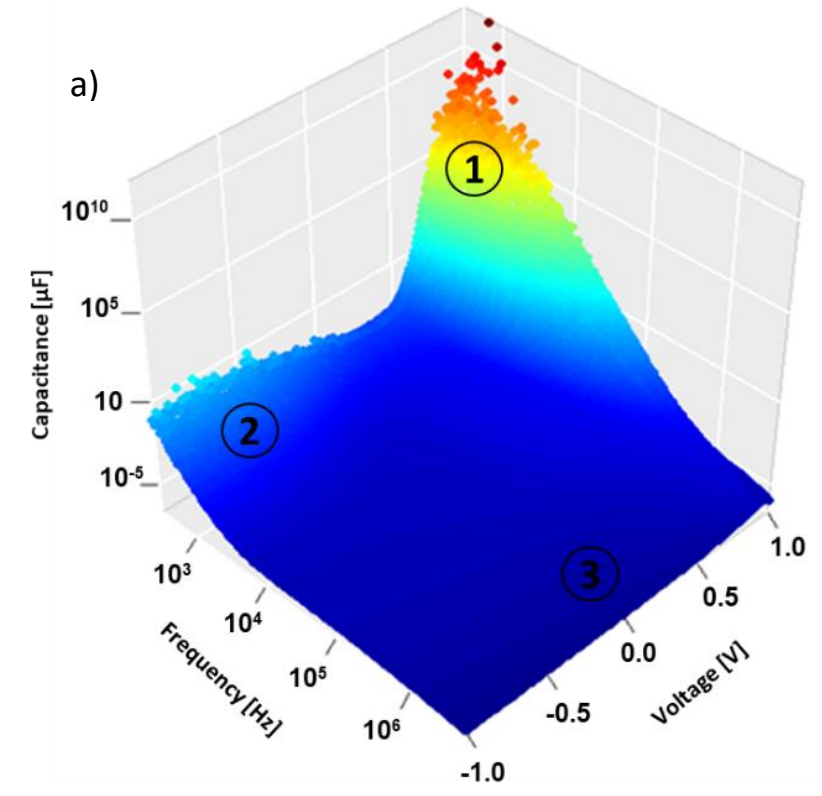

b) Neutral depletion region

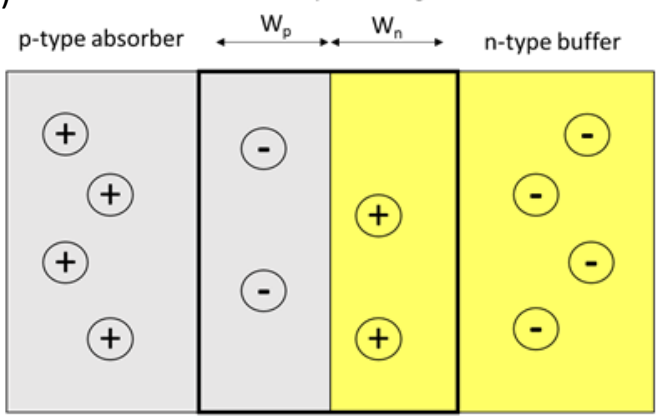

Electric field

Figure 6. a) Capacitance mapping from a RC circuit vs frequency and voltage, measured under dark, with the three mentioned regions marked and b) scheme of the charges in the p-n junction.

$$
C=\varepsilon_{0} \varepsilon_{r} \frac{A}{W}
$$

$C$ is the capacitance, $\varepsilon_{0}$ the vacuum permittivity, $\varepsilon_{r}$ the relative permittivity, $A$ is the area of the cell and $W$ the depletion width.

$$
C_{d}=I \frac{\tau_{T}}{V}=\frac{\tau_{T}}{R}
$$

$C_{d}$ is the diffusion capacitance, $I$ is the current, $\tau_{T}$ is the transit time, $V$ is the applied bias and $\mathrm{R}$ the equivalent resistance.

ii) When the applied bias is smaller than the built-in potential, the width of the depletion region is narrowed within the voltage, following the (Eq.3). Typically, the buffer layer required a highdensity carrier concentration $N_{d}>>N_{a}$, so most of the depletion width falls into the absorber and the equation can be simplified as in the (eq.4). At negative bias, the polarization is inverted and the current is blocked, thus the diffusion capacitance is negligible and the capacitance is dominated by the charge drift. 


$$
W_{p \vee n}=\sqrt{\frac{2 \varepsilon_{0} \varepsilon_{s}}{q\left(N_{d}+N_{a}\right)} \frac{N_{d \vee a}}{N_{a \vee d}}\left(V_{b}-V\right)}
$$

$W=W_{p}+W_{n}=\sqrt{\frac{2 \varepsilon_{0} \varepsilon_{S}}{q} \frac{N_{d}+N_{a}}{N_{a} N_{d}}\left(V_{b}-V\right)} \approx \sqrt{\frac{2 \varepsilon_{0} \varepsilon_{S}}{q N_{a}}\left(V_{b}-V\right)}$

$W_{p}$ and $W_{n}$ are the width of the depletion region in the $p$ and $n$ material respectively, $\varepsilon_{s}$ is the relative permittivity, $N_{d}$ is the donor concentration in the $\mathrm{n}$ material, $N_{a}$ is the acceptor concentration of the p material, $V_{b}$ is the built-in potential.

iii) At high frequency, the transit time $\tau_{T}$ is much larger than the signal and the charges has no sufficient time to transfer across the device, provoking a drop in the capacitance. At this region, the single effect remaining is the series resistance.

A solar cell is a complex circuit, where many simultaneous processes occurred, thus a simple RC circuit will not be able to provide reliable information. To further expand this, we made fitting in an equivalent circuit to the Nyquist plot. Nyquist plot vs voltage map (Fig. 7a) represents, semicircles that size changes with the voltage. These semicircles fits with equivalent circuit composed by a series resistance followed by a certain number of loops with a capacitor and a resistance ${ }^{36,37}$, as illustrated in the Fig. 7 b. Each loop represents a physical process occurring inside the devices. This part is very tricky, as some of the semicircles can be overlapped, making the analysis complex. The first semicircle is usually associated with the junction $\mathrm{p}^{+} / \mathrm{p}$ in the back contact with the $\operatorname{MoS}(e)_{2}$, while the main semicircle is associated with the $p-n$ junction. Other processes as trap states or grain boundaries is generally overlapped with the main semicircle, producing a modification in the shape. These semicircles are ideally described as the scheme showed in Fig.7c. By minimizing the error between the equivalent circuit and the measurement, different values of the capacitance and resistance can be extracted for different processes, independently from the frequency.

a)
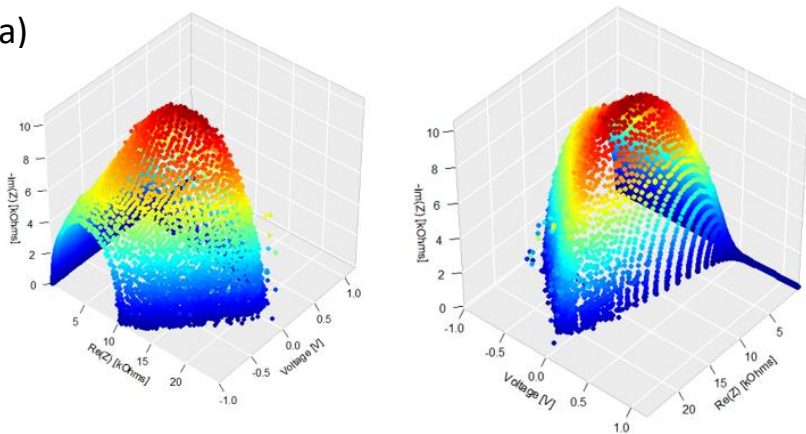

b)

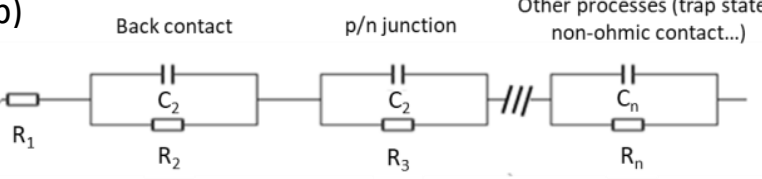

c)

$$
Z(f)=R_{1}+\sum_{i=2}^{n} \frac{R_{i}}{1+i 2 \pi f R_{i} C_{i}}
$$

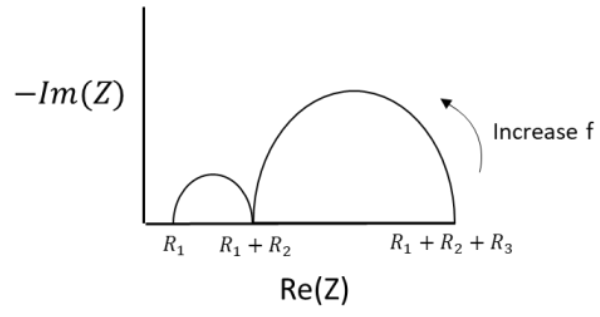

Figure 7. a) Map of the Nyquist plot vs bias voltage with different view perspectives measured in dark, b) equivalent circuit used to fitting the solar cells and c) equation that describes the equivalent circuit for $n$ loops and representation scheme for $n=3$.

From the value of the capacitance $C_{3}$, which represent $p-n$ junction, a Mott-Schottky plot can be determined by plotting $\left(\text { Area } / C_{3}\right)^{2}$ vs applied voltage. Fig.8a illustrates the MottSchottky of the CZTS samples when a $\mathrm{CZTS} / \mathrm{C}_{60} / \mathrm{CdS}$ and a CZTS/CdS/ $\mathrm{C}_{60}$ configuration is used. Typically, the built-in voltage can be calculated by the intercepting the $x$ axis to a linear fit at voltage lower than $\mathrm{V}_{\mathrm{B}}$, but in this case it does not follow a linear trend, and fits well with a second order polynomial. This is a characteristic behaviour of non-crystaline materials, which indicates the presence of non-ideal processes such as deep levels. Therefore, the built-in potencial is calculated by the extrapolation of the polynomial fit to the cross with the $\mathrm{x}$ axis, and a term of $k T / q$ is substracted. We can deduct from Table 3 a significant increase in the built-in voltage of $155 \mathrm{mV}$ for the sample CZTS/ $\mathrm{C}_{60} / \mathrm{CdS}$, pointing towards the increase of $V_{o c}$ in these solar cells.

By elucidating the $\boldsymbol{V}_{\boldsymbol{b} \boldsymbol{i}}$ value, a representation of the donor concentration can be calculated by the (Eq.5), using the same criteria $N_{A} \gg N_{D}$. Donor concentration vs depletion width is represented in the Fig. $8 b$, where the width is calculated by the (Eq.1). It can be noted that the depletion width is deeper and the donor density is lower for the CZTS/CdS/ $\mathrm{C}_{60}$ sample. This is a complimentary situation, as the electron-holes pairs can be separated deeper in the absorber material, but a low life time of the minority carriers in the absorber can produce a negative effect in the collection. The $\mathrm{C}_{60}$ employed as buffer layer will passivate CZTS surface, which in turn prevent surface recombination and therefore the hole concentration is increased.

$$
\begin{gathered}
\frac{A^{2}}{C^{2}}=\frac{2}{q \varepsilon_{0} \varepsilon_{\text {buffer }} \varepsilon_{C Z T S}} \frac{N_{D} \varepsilon_{\text {buffer }}+N_{A} \varepsilon_{C Z T S}}{N_{D} N_{A}}\left(V_{b}-V\right) \\
\stackrel{N_{A} \gg N_{D}}{\approx} \frac{2}{q \varepsilon_{0} \varepsilon_{C Z T S}} \frac{V_{b}-V}{N_{D}}
\end{gathered}
$$

Table 3. Calculated parameters from $C-V$ measurements at $V=0$, in dark

\begin{tabular}{ccccc}
$\begin{array}{c}\text { VALUES FOR } \\
\text { V }=0\end{array}$ & $\boldsymbol{V}_{\boldsymbol{b i}}(\boldsymbol{V})$ & $\boldsymbol{N}_{\boldsymbol{D}}\left(\mathbf{c m}^{-3}\right)$ & $\boldsymbol{W}(\boldsymbol{n m})$ & $\begin{array}{c}\boldsymbol{\tau}_{\boldsymbol{T}} \\
(\mathrm{NS})\end{array}$ \\
\hline $\mathrm{CZTS} / \mathrm{CdS} / \mathrm{C}_{60}$ & 0.709 & $1.41 \times 10^{16}$ & 183 & 555 \\
$\mathrm{CZTS} / \mathrm{C}_{60} / \mathrm{CdS}$ & 0.864 & $3.47 \times 10^{16}$ & 128 & 104.5
\end{tabular}



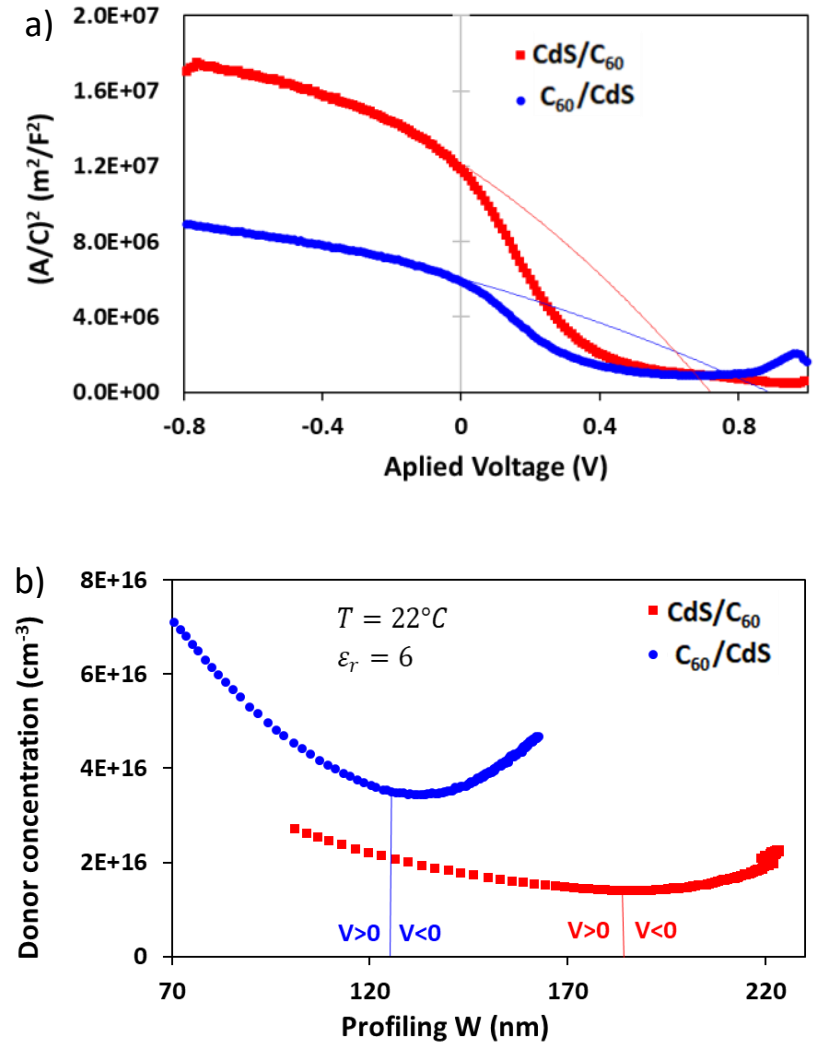

Figure 8. a) Mott-Schottky plot with a polynomic fit of the negative voltage and b) profiling of the calculated donor concentration.

The transit time $\left(\tau_{T}\right)$ was calculated by multiplying $C_{3}$ and $R_{3}$ of the equivalent circuit, and is represented vs the voltage (Fig. 9a) and the characteristic values of both cells are showed in the Table 2. Longer transit times suggests higher charge accumulation at the interface, and thus less efficient charge extraction. The transit time for the devices with $\mathrm{CZTS} / \mathrm{C}_{60} / \mathrm{CdS}$ is around five times shorter than of $\mathrm{CZTS} / \mathrm{CdS} / \mathrm{C}_{60}$. This notable longer time for the $\mathrm{CZTS} / \mathrm{CdS} / \mathrm{C}_{60}$ sample can explain the loss of current measured, as the significant part of the accumulated charge will have tendency to recombine before its extraction. To complement our experiments, for the sample CZTS/ $\mathrm{C}_{60} / \mathrm{CdS}$ sample, the impedance spectra were also measured and compared under light illumination near to $V_{\text {oc }}\left(700 \mathrm{mV} V_{o c}\right)$ and under the equivalent bias voltage of $700 \mathrm{mV}$ (Fig.9b). It can be noted that the shape of semicircle decreases under illumination, due to decrease in both the resistance and the capacitance. At this point, the carrier diffusion dominates, but the transit time value decreases substantially. This result reaffirms that the $\mathrm{C}_{60} / \mathrm{CdS}$ buffer layer is highly photoactive. This effect also produces that the power extracted from a solar cell can be further increase with the illumination time. Measurements following the maximum power point under 1 sun illumination (Fig.S4) shows that during the first 30-40 s the observed power increase is mainly due to an increase in the extracted current.

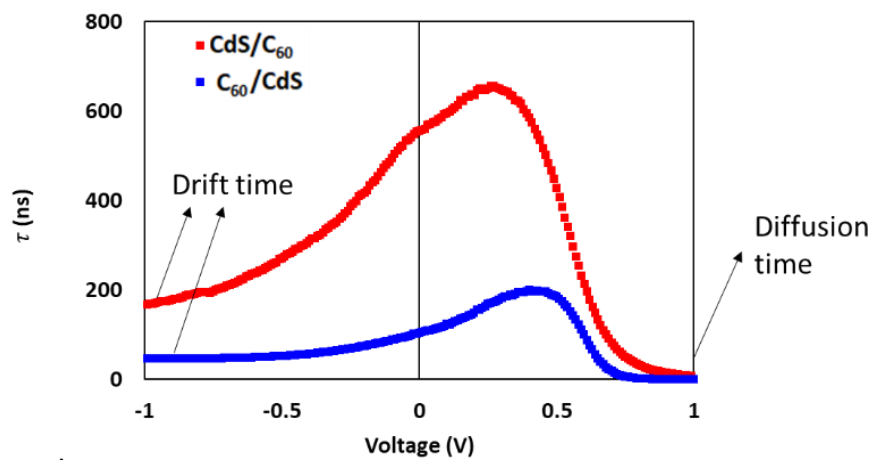

b)

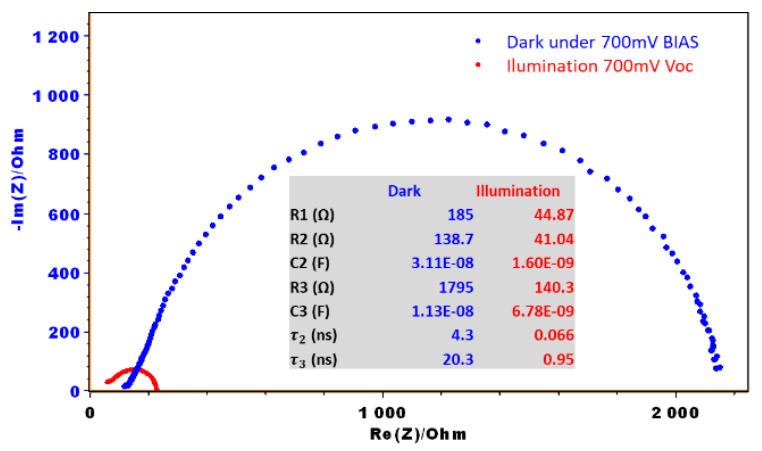

Figure 9. a) Transit time at different applied bias and b) impedance spectra under illumination (white LED), having $700 \mathrm{mV}$ of $V_{\mathrm{oc}}$, and in dark on an applied bias of $700 \mathrm{mV}$. The parameters of fit with $n=3$ are showed in the table inside.

\section{Conclusions}

The partial substitution of CdS by an organic charge transport materials is shown to be an effective method to reduce the loading of $\mathrm{Cd}$ based materials, without at the cost of device perfomance in the solar cells. We noted that reducing the thickness of CdS also induce an increase in photocurrent in the violet and UV region of the spectra, region where the absorption of CdS is parasitic. The employed organic charge transport laters was noted to be stable under the required post treatment temperatures of 240 ㅇ C for fabrication of solar cells. Among its derivatives, $\mathrm{C}_{60}$ was found to show better electrooptical properties than $C_{70}$ as buffer layer in a kesterites solar cell. For pure sulfide CZTS kesterite, the use of a double buffer of $\mathrm{C}_{60}$ and $\mathrm{CdS}$ was noted to reduce the interfacial recombination that usually plague the CZTS/CdS interface.

One of the main barriers in inorganic solar cells is the limited choice of materials that can be used efficiently as buffer layers, and is being typically dependent on cadmium or indium elements. Organic charge transport materials having ease of flexilblity and choice of core groups to tune the electro-optical properties, and are solution processable. Our investigation paves the way for further optimization and exploration of employing organic materials for kesterite type solar cells to develop cost effective and green photovoltaics.

\section{Conflicts of interest}

There are no conflicts to declare. 


\section{Acknowledgements}

This work is possible thanks to the funding from the Basque Country PI2018-08 (PISCES) and supported by H2020 European Research Council Grant (MOLEMAT, 72630), which are gratefully acknowledge. The Ministry of Science and Innovation of Spain under IGNITE project (ENE2017-87671-C3-1-R), the European Regional Development Funds (ERDF, FEDER Programa Competitivitat de Catalunya 20072013) and CERCA Programme / Generalitat de Catalunya, Authors from IREC belong to the SEMS (Solar Energy Materials and Systems) Consolidated Research Group of the "Generalitat de Catalunya" (Ref. 2017 SGR 862).

\section{References}

1 S. Giraldo, Z. Jehl, M. Placidi, V. Izquierdo-Roca, A. PérezRodríguez and E. Saucedo, Adv. Mater., 2019, 1806692.

2 T. Todorov, H. W. Hillhouse, S. Aazou, Z. Sekkat, O. Vigil-Galán, S. D. Deshmukh, R. Agrawal, S. Bourdais, M. Valdés, P. Arnou, D. B. Mitzi and P. J. Dale, J. Phys. Energy, 2020, 2, 012003

3 L. H. Wong, A. Zakutayev, J. D. Major, X. Hao, A. Walsh, T. K. Todorov and E. Saucedo, J. Phys. Energy, 2019, 1, 032001.

4 C. Platzer-Björkman, N. Barreau, M. Bär, L. Choubrac, L. Grenet, J. Heo, T. Kubart, A. Mittiga, Y. Sanchez, J. Scragg, S. Sinha and M. Valentini, J. Phys. Energy, 2019, 1, 044005.

5 O. Vigil-Galán, F. A. A. Pulgarín, F. Cruz-Gandarilla, M. Courel, G. Villarreal-Ruiz, Y. Sánchez, D. Jiménez-Olarte and E. Saucedo, Mater. Des., 2016, 99, 254-261.

6 M. Neuschitzer, Y. Sanchez, S. Lopez-Marino, H. Xie, A. Fairbrother, M. Placidi, S. Haass, V. Izquierdo-Roca, A. PerezRodriguez and E. Saucedo, Prog. Photovoltaics Res. Appl. 2015, 23, 1660-1667.

7 M. Nguyen, K. Ernits, K. F. Tai, C. F. Ng, S. S. Pramana, W. A. Sasangka, S. K. Batabyal, T. Holopainen, D. Meissner, A. Neisser and L. H.Wong, Sol. Energy, 2015, 111, 344-349.

8 A. Ghosh, R. Thangavel and A. Gupta, J. Alloys Compd., 2017, 694, 394-400.

9 M. Neuschitzer, K. Lienau, M. Guc, L. C. Barrio, S. Haass, J. M. Prieto, Y. Sanchez, M. Espindola-Rodriguez, Y. Romanyuk, A. Perez-Rodriguez, V. Izquierdo-Roca and E. Saucedo, J. Phys. D. Appl. Phys., 2016, 49, 125602.

10 D. A. R. Barkhouse, R. Haight, N. Sakai, H. Hiroi, H. Sugimoto and D. B. Mitzi, Appl. Phys. Lett., 2012, 100, 193904

11 T. Ericson, F. Larsson, T. Törndahl, C. Frisk, J. Larsen, V. Kosyak, C. Hägglund, S. Li and C. Platzer-Björkman, Sol. RRL, 2017, 1 1700001.

12 K. Sun, C. Yan, F. Liu, J. Huang, F. Zhou, J. A. Stride, M. Green and X. Hao, Adv. Energy Mater., 2016, 6, 1600046.

13 H. Hiroi, J. Kim, M. Kuwahara, T. K. Todorov, D. Nair, M Hopstaken, Y. Zhu, O. Gunawan, D. B. Mitzi and H. Sugimoto, in 2014 IEEE 40th Photovoltaic Specialist Conference, PVSC 2014, IEEE, 2014, pp. 30-32.

$14 \mathrm{H}$. W. Kroto, J. R. Heath, S. C. O'Brien, R. F. Curl and R. E. Smalley, Nature, 1985, 318, 162-163.

15 N. S. Sariciftci, D. Braun, C. Zhang, V. I. Srdanov, A. J. Heeger, G. Stucky and F. Wudl, Appl. Phys. Lett., 1993, 62, 585-587.

16 M. Koltun, D. Faiman, S. Goren, E. A. Katz, E. Kunoff, A. Shames, S. Shtutina and B. Uzan, Sol. Energy Mater. Sol. Cells, 1996, 44, 485-491.

17 C.-H. Chiang and C.-G. Wu, Nat. Photonics, 2016, 10, 196-200.

18 K. Wojciechowski, T. Leijtens, S. Siprova, C. Schlueter, M. T. Hörantner, J. T. W. Wang, C. Z. Li, A. K. Y. Jen, T. L. Lee and H. J. Snaith, J. Phys. Chem. Lett., 2015, 6, 2399-2405.
19 C. Chen, S. Zhang, S. Wu, W. Zhang, H. Zhu, Z. Xiong, Y. Zhang and W. Chen, RSC Adv., 2017, 7, 35819-35826.

20 J.-J. Wang, J.-S. Hu, Y.-G. Guo and L.-J. Wan, NPG Asia Mater., 2012, 4, e2-e2.

21 S. J. Jang, N. T. Ho, M. H. Lee and Y. S. Kim, in Journal of Physics: Conference Series, IOP Publishing, 2017, vol. 864, p. 012065.

22 S. K. Saha, A. Guchhait and A. J. Pal, Phys. Chem. Chem. Phys., 2012, 14, 8090-8096.

23 S. Kim, K. M. Kim, H. Tampo, H. Shibata and S. Niki, Appl. Phys. Express, 2016, 9, 102301

24 A. V. Shamardin, A. S. Opanasyuk, D. I. Kurbatov and M. E. Istratov, in Proceedings of the 2017 IEEE 7th International Conference on Nanomaterials: Applications and Properties, NAP 2017, Institute of Electrical and Electronics Engineers Inc., 2017, vol. 2017-January.

25 M. Neuschitzer, Y. Sanchez, S. Lõpez-Marino, H. Xie, A Fairbrother, M. Placidi, S. Haass, V. Izquierdo-Roca, A. PerezRodriguez and E. Saucedo, Prog. Photovoltaics Res. Appl., 2015, 23, 1660-1667.

26 K. Mokurala, L. L. Baranowski, F. W. de Souza Lucas, S. Siol, M. F. A M van Hest, S. Mallick, P. Bhargava and A. Zakutayev, , DOI:10.1021/acscombsci.6b00074.

27 S. Lee, E. S. Lee, T. Y. Kim, J. S. Cho, Y. J. Eo, J. H. Yun and A. Cho, Sol. Energy Mater. Sol. Cells, 2015, 141, 299-308.

28 W.-C. Chen, C.-Y. Chen, Y.-R. Lin, J.-K. Chang, C.-H. Chen, Y.-P. Chiu, C.-I. Wu, K.-H. Chen and L.-C. Chen, Mater. Today Energy, 2019, 13, 256-266.

29 V. I. Zubov, N. P. Tretiakov, J. N. Teixeira Rabelo and J. F. Sanchez Ortiz, Phys. Lett. A, 1994, 194, 223-227.

30 H. S. Lin, I. Jeon, R. Xiang, S. Seo, J. W. Lee, C. Li, A. Pal, S. Manzhos, M. S. Goorsky, Y. Yang, S. Maruyama and Y. Matsuo, ACS Appl. Mater. Interfaces, 2018, 10, 39590-39598.

31 M. Bär, B. A. Schubert, B. Marsen, R. G. Wilks, S. Pookpanratana, M. Blum, S. Krause, T. Unold, W. Yang, L. Weinhardt, C. Heske and H. W. Schock, Appl. Phys. Lett., 2011, 99, 222105

32 A. Fairbrother, E. García-Hemme, V. Izquierdo-Roca, X Fontané, F. A. Pulgarín-Agudelo, O. Vigil-Galán, A. PérezRodríguez and E. Saucedo, J. Am. Chem. Soc., 2012, 134 8018-8021.

33 H. Sun, K. Sun, J. Huang, C. Yan, F. Liu, J. Park, A. Pu, J. A. Stride, M. A. Green and X. Hao, ACS Appl. Energy Mater., 2018, 1, 154-160.

34 H. Xie, Y. Sáncheza, P. Tang, M. Espíndola-Rodríguez, M. Guc, L. Calvo-Barrio, S. López-Marino, Y. Liu, J. R. Morante, A. Cabot, V. Izquierdo-Roca, J. Arbiol, A. Pérez-Rodríguez and E. Saucedo, Sol. RRL, 2018, 3, 1800279.

35 C. Yan, J. Huang, K. Sun, S. Johnston, Y. Zhang, H. Sun, A. Pu, M. He, F. Liu, K. Eder, L. Yang, J. M. Cairney, N. J. Ekins-Daukes, Z. Hameiri, J. A. Stride, S. Chen, M. A. Green and X. Hao, Nat. Energy, 2018, 3, 764-772.

36 P. A. Fernandes, P. M. P. Salomé, A. F. Sartori, J. Malaquias, A. F. Da Cunha, B. A. Schubert, J. C. González and G. M. Ribeiro, Sol. Energy Mater. Sol. Cells, 2013, 115, 157-165.

37 G. K. Gupta, A. Garg and A. Dixit, J. Appl. Phys., DOI:10.1063/1.5002619. 